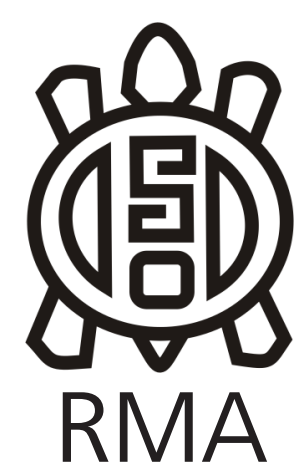

Arqueología

\title{
Pinturas rupestres y comunicación en la cuenca de los lagos Tar y San Martin (provincia de Santa Cruz)
}

\author{
Rock paintings and communication in the Tar and San Martin lakes basin \\ (Santa Cruz province)
}

\author{
Anahí Re* y Juan Bautista Belardi**
}

*Anahí Re. Instituto Nacional de Antropología y Pensamiento Latinoamericano, CONICET, Universidad de Buenos Aires, Argentina. E-mail: anahire1@gmail.com **Juan Bautista Belardi. Universidad Nacional de la Patagonia Austral, ICASUR, Unidad Académica Río Gallegos, CONICET, Argentina. E-mail: juanbautistabelardi@gmail.com

\begin{abstract}
Resumen
Se aborda el análisis de las representaciones rupestres de los sitios Bloque 1 del Campo de Bloques 1 y Alero con Manos del Río Chalía, relevados en los sectores bajos asociados a la cuenca de los lagos Tar y San Martín (sudoeste de la provincia de Santa (ruz). Aquí se resumen las particularidades de ambos sitios y se propone una secuencia de ejecución de los motivos. Asimismo, los sitios son contextualizados con la información general sobre uso del espacio regional y con los datos disponibles sobre otros sitios con representaciones rupestres ubicados en las cercanías y a distintas altitudes. El análisis indica que en los sectores bajos asociados a la cuenca de los lagos Tar y San Martín se evidencia una baja densidad de representaciones rupestres, el uso exclusivo de la pintura y una baja diversidad de motivos que se diferencia de lo observado en espacios lindantes. De este modo, el estudio integrado de estas representaciones permite reconocer particularidades de los sectores bajos y evaluar la jerarquización de los espacios y la dinámica de circulación de información, principalmente durante el Holoceno tardío.
\end{abstract}

Palabras clave: Representaciones rupestres; Cuencas bajas; Circulación de información; Holoceno tardío; Patagonia meridional.

\begin{abstract}
The study of the painted rock art from Bloque 1 del Campo de Bloques 1 and Alero con Manos del Río Chalía is undertaken. Both were documented in lowlands associated to the Tar and San Martin lakes basin (South-Western Santa Cruz province). The characteristics of the sites and the rock art production sequence are summarized. Sites are contextualized within general data on regional landscape use as well as with the information from other rock art sites located at different altitudes in nearby regions. The analysis indicates a low density of rock art motifs, together with the exclusive use of painting and a low diversity of motifs in the lowlands associated with the Tar and San Martin lakes basin. All these characteristics differ from adjoining areas. Hence, the study of this rock art enables the identification of particularities of the lowlands and the evaluation of, the ranking of these areas and the dynamic of information circulation in the past.
\end{abstract}

Keywords: Rock art; Lowland basins; Information exchange; Late Holocene; Southern Patagonia.

Se abordan las características de las representaciones rupestres relevadas en los sectores bajos (270-400 msnm) asociados a la cuenca de los lagos Tar y San Martín (sudoeste de la provincia de Santa Cruz). Hasta el momento se han registrado dos sitios con pinturas rupestres, que presentan características contrastantes. Uno de ellos es el Bloque 1 del Campo de Bloques 1, ubicado en el extremo sureste de la cuenca del lago San Martín, el cual presenta un único motivo. El segundo es el Alero con Manos del Río Chalía, que tiene más de 280 motivos y que se encuentra en la vía de acceso natural a los lagos desde el este, en la cuenca superior del río homónimo. Se posiciona a unos $65 \mathrm{~km}$ hacia el este del Bloque 1 y tiene más de 280 motivos. Éste se encuentra a unos $13 \mathrm{~km}$ de la actual localidad de Tres Lagos. De esta manera, el primer objetivo del trabajo es analizar las representaciones registradas a partir de diferentes variables (ubicación, tipos de motivos, técnicas, tonalidades, superposiciones y estado de conservación). A partir de la información presentada, se postula una secuencia de producción de las pinturas.

Luego, se realiza una contextualización de los sitios analizados con la información disponible sobre uso del espacio en la cuenca bajo estudio, lo que permite proponer una asignación cronológica tentativa para las representaciones. De esta forma, se integran los motivos rupestres con los datos que han sido generados a partir de otras líneas de evidencia. Por último, se plantea la necesidad de considerar los dos sitios en conjunto con otros documentados en regiones cercanas. Entre ellos se cuentan sitios en espacios altos -por sobre los 700 
msnm- como las mesetas de San Adolfo y Cardiel Chico, del Tobiano y Strobel. También se incluyen otros ubicados en altitudes intermedias sobre la margen norte del lago Viedma, las nacientes del río Chalía y en cercanías de la laguna El Pajonal (en campos entre los lagos Tar y Viedma). Por último, se comparan sitios por debajo de la cota 400 msnm como los registrados en Ea. La Margarita (noreste del lago Viedma) y en la cuenca del lago Cardiel (Figura 1). El estudio integrado de las representaciones rupestres permite reconocer particularidades de los sectores bajos que deben ser comprendidas para así evaluar la dinámica de circulación de información en el pasado.

\section{Motivos rupestres y comunicación}

Los motivos rupestres son considerados artefactos y por lo tanto un componente más del registro arqueológico (Aschero 1988). Así, pueden ser estudiados desde marcos espaciales y temporales amplios modelando sus distribuciones. Asimismo, se entiende que los motivos rupestres fueron utilizados para comunicar información de distinto tipo y que por lo tanto pueden tener un valor adaptativo (Barton et al. 1994; McDonald y Veth 2011; Belardi et al. 2016a, entre otros). De esta forma, las variaciones temporales y espaciales en la frecuencia y motivos de representaciones pueden ser usadas para discutir el flujo de información en diferentes escalas (Barton et al. 1994). Otro aspecto importante de dichas consideraciones es que la realización de los motivos rupestres implica la modificación intencional del ambiente. Así, junto con su papel comunicacional, conforman una parte de la construcción de nicho de las poblaciones humanas (sensu Aunger 2009).

Esto permite discutir sobre una base arqueológica la interacción entre poblaciones, entendida como el intercambio de materiales, ideas, creencias e información entre miembros de diferentes grupos corporativos (Odess 1998: 417). Si se considera que las similitudes pueden ser producto de relaciones históricas, se establece un puente analítico entre distribuciones de motivos de determinadas características definidas por el investigador y la comunicación de información, con independencia del conocimiento que se pueda llegar a tener sobre el contenido o significado del mensaje para aquellos que establecieron el nexo comunicacional. Esto se refuerza si se cuenta con cronologías independientes que vinculen similitudes entre distintos conjuntos de artefactos que permitan comparar con las tendencias observadas en otras líneas de evidencia, como la circulación de materias primas líticas de proveniencia conocida e información biológica humana (por ejemplo, isotópica, genética, deformaciones intencionales y tratamiento del cuerpo).

\section{La región de estudio}

La cuenca de los lagos Tar y San Martín se encuentra en el sudoeste de la provincia de Santa Cruz a $49^{\circ} 7^{\prime}$ Latitud Sur y $72^{\circ} 11^{\prime}$ Longitud Oeste, a aproximadamente 300 msnm (Figura 1). Cabe aclarar que el lago San Martín continúa hacia Chile con el nombre de lago O’Higgins. La cuenca tiene una orientación noroeste-sudeste y está delimitada al oeste por el Campo de Hielo Patagónico Sur, hacia el norte por las mesetas de San Adolfo y Cardiel Chico y hacia el sur por las mesetas y pampas que la comunican con el lago Viedma.

El paisaje ha sido modelado por la acción glaciaria, así, se identifican diferentes sistemas de morrenas (Rabassa y Coronato 2002). Mientras que el lago Tar se presenta más abierto, el San Martín se encuentra encajonado entre mesetas altas y la cordillera de los Andes. Un importante gradiente de precipitaciones decrece desde los $800 \mathrm{~mm}$ en el oeste hasta menos de $200 \mathrm{~mm}$ en el este. Esto conlleva una diferenciación ambiental que incluye: 1) bosque y ecotono bosque-estepa en el oeste del lago San Martín, caracterizados por una estacionalidad más marcada y 2) estepa graminosa al este del lago y 3) semidesierto y estepa arbustiva y subarbustiva hacia el este en los alrededores del lago Tar y del cerro Cach Aike (Bamonte et al. 2013).

A partir de análisis polínicos y de partículas de carbón sedimentario depositado en secuencias locales, se han planteado variaciones ambientales en las cuales se alternan períodos más secos y más húmedos a lo largo del Holoceno (Bamonte et al. 2013; Belardi et al. 2013a). El escenario actual se establecería aproximadamente hace 3000 años. A partir de ese momento, en función del cambio en la dirección e intensidad de los vientos, se produciría la amplia diversidad interna en las precipitaciones y la vegetación que se evidencia hoy en día.

La cuenca de los lagos Tar y San Martín linda hacia el sur y este con el curso superior del río Chalía. Se destaca que el valle de este río es un eje de circulación natural que atraviesa la provincia de Santa Cruz de oeste a este. Por otra parte, la cuenca también está próxima al eje de circulación norte-sur que coincide con el actual trazado de la Ruta Nacional 40.

\section{Las investigaciones arqueológicas}

Las investigaciones sistemáticas en las cuenca de los lagos Tar y San Martín se iniciaron en 2006 y se focalizaron en comprender la dinámica cambiante del poblamiento humano a lo largo del Holoceno (Belardi et al. 2009, 2010, 2013a, 2013b; Belardi y Carballo Marina 2014; Espinosa et al. 2007, 2013, entre otros). Los trabajos, que han tenido una perspectiva distribucional, han incluido la realización de prospecciones, transectas, sondeos y excavaciones. En su mayoría se han centralizado en el estudio de la tecnología lítica y de los restos arqueofaunísticos. Se realizaron además aportes desde la bioarqueología, los isótopos estables y los estudios 


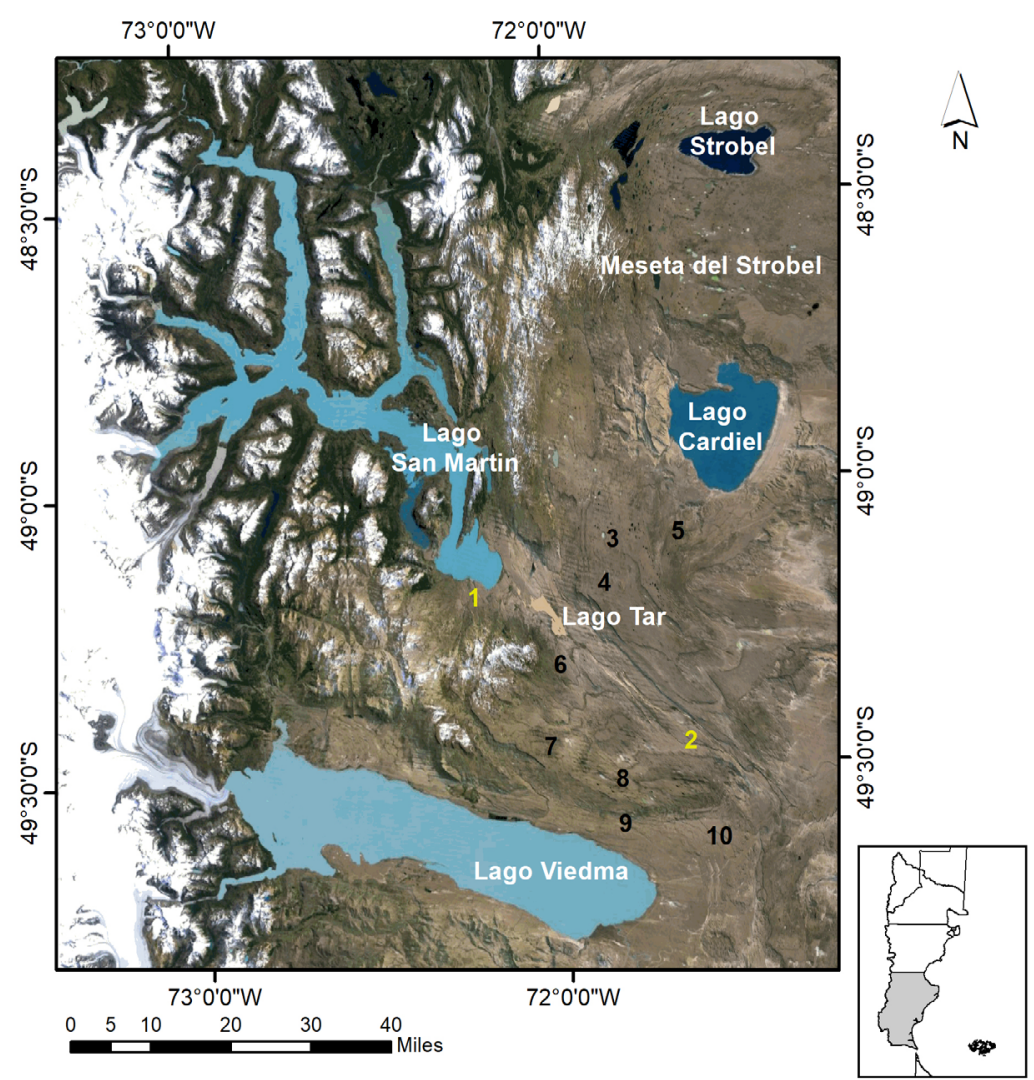

Figura 1. Ubicación de la cuenca de los lagos Tar y San Martín y sitios y regiones mencionados en el texto. Referencias: 1: CB1B1, 2: AMRC, 3: Meseta del Cardiel Chico, 4: Meseta de San Adolfo, 5: La Criolla, 6: Nacientes del río Chalía, 7: Laguna El Pajonal, 8: Meseta del Tobiano, 9: Margen norte del lago Viedma, 10: La Margarita.

Figure 1. Location of the Tar and San Martin lakes basin and sites and regions mentioned in the text. References: 1: CB1B1, 2: AMRC, 3: Cardiel Chico Plateau, 4: San Adolfo Plateau, 5: La Criolla, 6: Chalía River Source, 7: El Pajonal Lagoon, 8: Tobiano Plateau, 9: North Bank of Viedma Lake, 10: La Margarita. polínicos. Por otra parte, en los sectores bajos de la cuenca también se localizaron sitios con representaciones rupestres, en particular pinturas, las cuales hasta aquí sólo habían sido mencionadas de manera muy sucinta (ver Belardi et al. 2010).

Para facilitar la comparación del registro arqueológico al interior de la cuenca se definieron tres sectores altitudinales: bajos (entre los 270 y los $400 \mathrm{msnm}$ ), intermedios (400 a 700 msnm) y altos (más de 700 msnm). Mientras que los primeros trabajos abordaron los alrededores del lago Tar y la margen sur del lago San Martín (Belardi et al. 2009, 2010; Belardi y Campan 2009; Espinosa et al. 2009), recientemente las investigaciones se focalizaron en el ecotono y bosque ubicados más al oeste (Belardi y Carballo Marina 2014; Espinosa et al. 2013).

Los 26 fechados radiocarbónicos disponibles indican que las ocupaciones se iniciaron hace ca. 11.100 años cal. AP y se intensificaron durante el Holoceno tardío hasta llegar a momentos históricos. De esta manera, 14 dataciones se concentran en los últimos 3000 años (Belardi et al. 2013a). Estos fechados serán más adelante considerados para proponer la asignación temporal de las representaciones rupestres analizadas.

El estudio de las evidencias arqueológicas ha llevado a proponer un uso diferencial al interior de la cuenca de los lagos Tar y San Martín que se habría mantenido en el tiempo. En primer lugar, la margen este del lago Tar y los alrededores del Cerro Cach Aike habrían tenido un uso más intenso de carácter residencial, articulando la comunicación y la circulación de bienes en una escala espacial amplia. Esto se propuso sobre la base de que los valles de los ríos Tar y Meseta, que desembocan en el lago Tar, posicionan al lago y, en particular, a su margen este como un punto intermedio entre las mesetas de San Adolfo y Cardiel Chico (al norte) y el lago Viedma (al sur). Ambos valles constituyen caminos de menor costo para la circulación en sentido latitudinal, posibilitando la articulación con las mesetas altas y cuencas lacustres aledañas (Belardi et al. 2009). Por otra parte, la ocupación del lago San Martín habría sido más esporádica y principalmente logística. Esto se enfatizaría hacia el oeste del lago, con un marcado carácter marginal (sensu Borrero 2004) al ser un "fondo de saco" donde el tránsito se dificulta por el bosque, la topografía quebrada y la cercanía al Campo de Hielo Patagónico Sur (Belardi et al. 2010, 2013a).

En forma complementaria se desarrollaron investigaciones en las mesetas de San Adolfo y Cardiel Chico, ubicadas al norte entre los 750 y 1100 msnm (Figura 1). Se registraron concentraciones de material lítico y parapetos, además de una alta frecuencia de grabados rupestres. Se planteó que las poblaciones humanas habrían utilizado estas mesetas tanto con componentes logísticos como residenciales principalmente durante el Holoceno tardío. La presencia de grabados y materias primas como obsidiana, proveniente de la Pampa del Asador (Espinosa 
y Goñi 1999), y limolita, disponible en la margen oeste del lago Cardiel (Belardi et al. 2015), fueron considerados indicadores de un flujo de bienes e información a través de las mesetas siguiendo un eje norte-sur (Belardi et al. 2009, 2013b, 2016b; Re et al. 2013-2014). En uno de los caminos de acceso a la meseta del Cardiel Chico desde el norte cabe mencionar el hallazgo a ca. 800 msnm de dos sitios con grabados y pinturas, denominados La Criolla 1 y 2, en uno de cuyos reparos se presentan distintos tipos de motivos pintados y grabados superpuestos, situación que es muy poco frecuente en la región y cuyo análisis está en desarrollo.

Por otra parte, también se realizaron trabajos sobre la margen norte del lago Viedma y en la meseta del Tobiano (Figura 1) (Belardi et al. 2016b). En sectores intermedios (400 a $700 \mathrm{msnm}$ ) se registraron diversos sitios con conjuntos artefactuales y restos óseos, algunos de ellos con grabados y pinturas. Se obtuvieron siete fechados concentrados en su mayoría en los últimos 3000 años. El más conocido de estos sitios es Punta del Lago Viedma (Menghin 1957; Schobinger y Gradin 1985), luego denominado Punta del Lago 1, dada la presencia de otros sitios (Belardi et al. 2016b). Por otra parte, en la meseta del Tobiano, a más de 900 msnm, se observó la presencia de parapetos, artefactos líticos y grabados rupestres (Belardi et al. 2016b).

Por último, hacia el sureste recientemente se realizaron trabajos en la Estancia La Margarita, en proximidad al curso medio del arroyo Los Paisanos (Figura 1). Si bien esta información todavía se encuentra inédita, cabe mencionar la presencia de dos sitios con representaciones rupestres entre los 300 y 400 msnm, que posteriormente son considerados en la comparación.

\section{Resultados: las pinturas rupestres de la cuenca}

En los sectores bajos (entre los 270 y los 400 msnm) asociados a la cuenca de los lagos Tar y San Martín se localizaron dos sitios con pinturas rupestres que son caracterizados a continuación (Figura 1). Cabe destacar que, si bien en términos generales existe una escasa oferta de reparos rocosos, se identificaron otros soportes potenciales tanto en la margen norte como en la sur del lago San Martín, en los cuales no se registraron manifestaciones. De esta manera, la evidencia indica una distribución muy acotada de los motivos rupestres.

\section{Sitio Bloque 1 del Campo de Bloques 1} (CB1B1).
Se ubica en el fondo de cuenca en la margen sureste del lago San Martin a $350 \mathrm{msnm}$, a menos de $1 \mathrm{~km}$ de la costa del lago (Figura 1) y se encuentra sobre el límite actual entre la estepa arbustiva y la graminosa (Bamonte et al. 2013). El Campo de Bloques 1 es producto de la remoción en masa y de movimientos gravitacionales de rocas filonianas de composición dacítica y se extiende a lo largo de 1400 metros en sentido este-oeste. Se registraron ocho bloques con reparo y materiales líticos en superficie, todos con sedimentos potencialmente excavables (Belardi et al 2010).

El Bloque 1 es un gran bloque partido, en el cual se han realizado excavaciones en dos sectores (Oquedad y Alero). Así, se cuenta con una secuencia de ocupación que se inicia hace 11.100 años cal. AP y llega hasta 800 años cal. AP con un hiato de 6.000 años (Belardi et al. 2010, 2013a). El análisis de la tecnología lítica y arqueofaunística ha indicado un uso de tipo logístico y de baja intensidad a lo largo de toda la secuencia (Pallo 2009; Belardi et al. 2010). Cabe aclarar que no se observaron en estratigrafía evidencias de producción de pinturas.

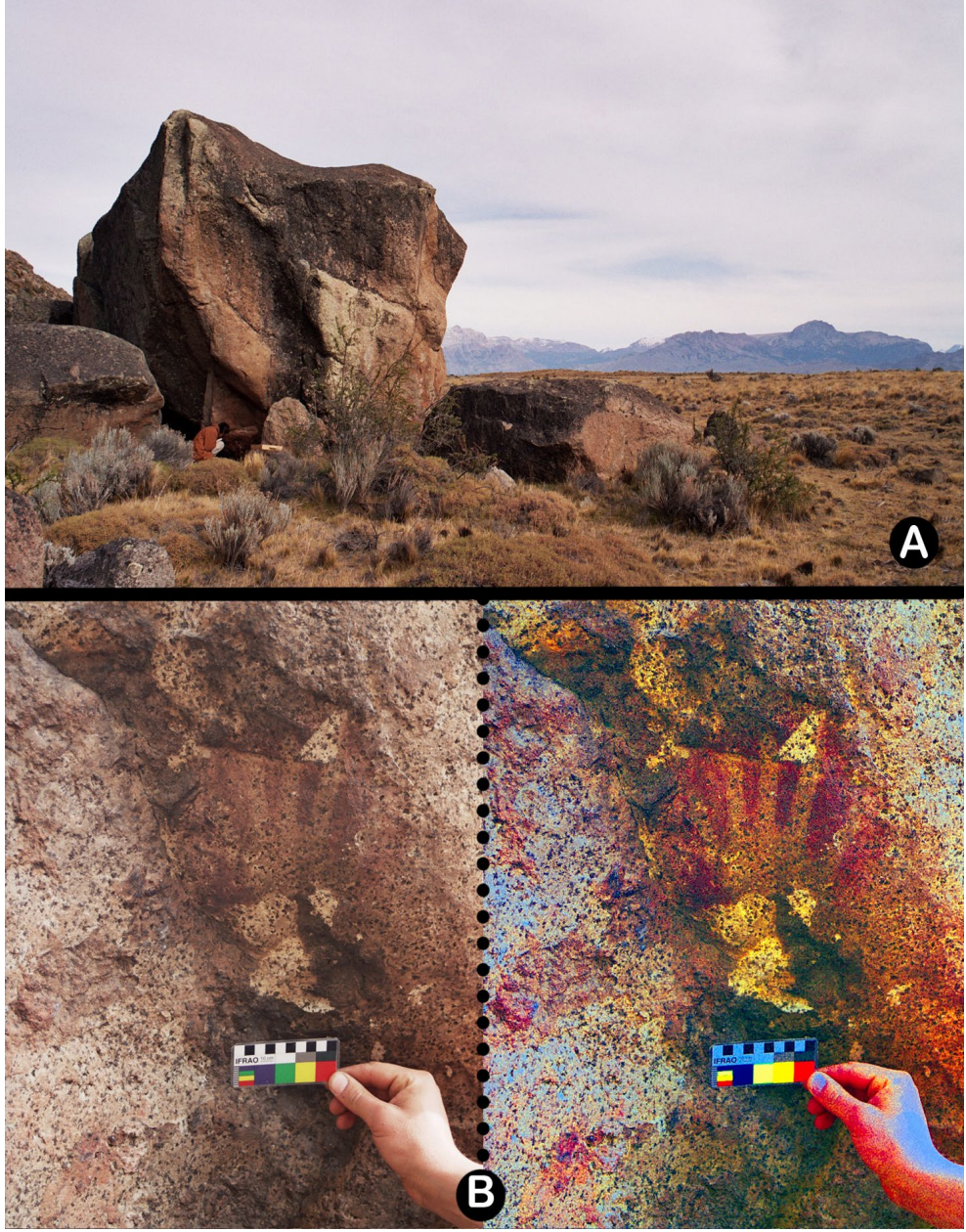

Figura 2. Bloque 1 del Campo de Bloques 1. A: Vista del bloque, B: Negativo de mano de CB1B1 (izquierda: foto original, derecha: foto con filtro DStretch).

Figure 2. Bloque 1 del Campo de Bloques 1. A: View of the boulder, B: Hand stencil in CB1B1 (left: original photograph, right: photograph with DStretch filter). 
En la cara sur de CB1B1 se ejecutó un único negativo de mano izquierda de tonalidad rojo violácea (Figura 2). Las dimensiones de la cara del bloque utilizada son 5 $\mathrm{m}$ de alto y 2,5 $\mathrm{m}$ de ancho. El negativo se encuentra a baja altura en un pequeño reparo y presenta muy baja visibilidad, dado su grado de desvaído y su ubicación. Su estado de conservación es regular, ya que el soporte se ha descascarado Al pie del motivo se realizó un sondeo que no arrojó materiales.

\section{Sitio Alero con Manos del Río Chalía (AMRC).}

Se encuentra a $300 \mathrm{msnm}$, en la estepa arbustiva y a $30 \mathrm{~km}$ al este del lago Tar (Figura 1), sobre el curso superior del río Chalía y está asociado a la principal vía de acceso desde el este a la cuenca bajo estudio. Se llega al sitio desde la Ruta Provincial 31 por un sendero de un kilómetro de extensión que bordea al río Chalía en su margen sureste. Dado su fácil acceso y escasa distancia (13 km), al día de hoy es visitado informalmente por los pobladores de la localidad de Tres Lagos.

El sitio tiene orientación norte y está sobre areniscas de la Fm. Piedra Clavada. Se observó material arqueológico tanto lítico como faunístico en la superficie de gran parte del paredón. No obstante, a diferencia de CB1B1, todavía no se dispone de información sobre estas líneas de evidencia. En cuanto a los motivos rupestres, se cuenta con relevamientos realizados en tres trabajos de campo (2007, 2009 y 2015), lo que ha permitido monitorear diferencias en el estado de preservación de las pinturas.

Las representaciones rupestres se distribuyen en un alero a lo largo de $50 \mathrm{~m}$ y en un nicho de 2,7 m de largo, 1,8 m de profundidad y $1 \mathrm{~m}$ de altura, ubicado $70 \mathrm{~m}$ al noroeste del primer abrigo (Figura 3).

El sitio en su conjunto posee 284 motivos, 264 en el alero propiamente dicho y 20 en el nicho (Tabla 1). A lo largo de todo el primero se presentan de forma bastante continua y allí donde se interrumpen se observan caídas de agua y bloques desprendidos, por lo cual se entiende que muy probablemente esos espacios también tuvieran pinturas. Para el relevamiento, el alero de $50 \mathrm{~m}$ de largo se dividió en cuatro sectores definidos por quiebres en la roca de caja. Si bien los sectores tienen distinta longitud (Tabla 1), si se toman en conjunto la cantidad de motivos registrados y el largo de cada sector, se observa una densidad similar a lo largo de todo el sitio (Tabla 1). Por otra parte, cabe destacar que las pinturas llegan a alcanzar alturas máximas de 3,15 m en el Sector 4 .

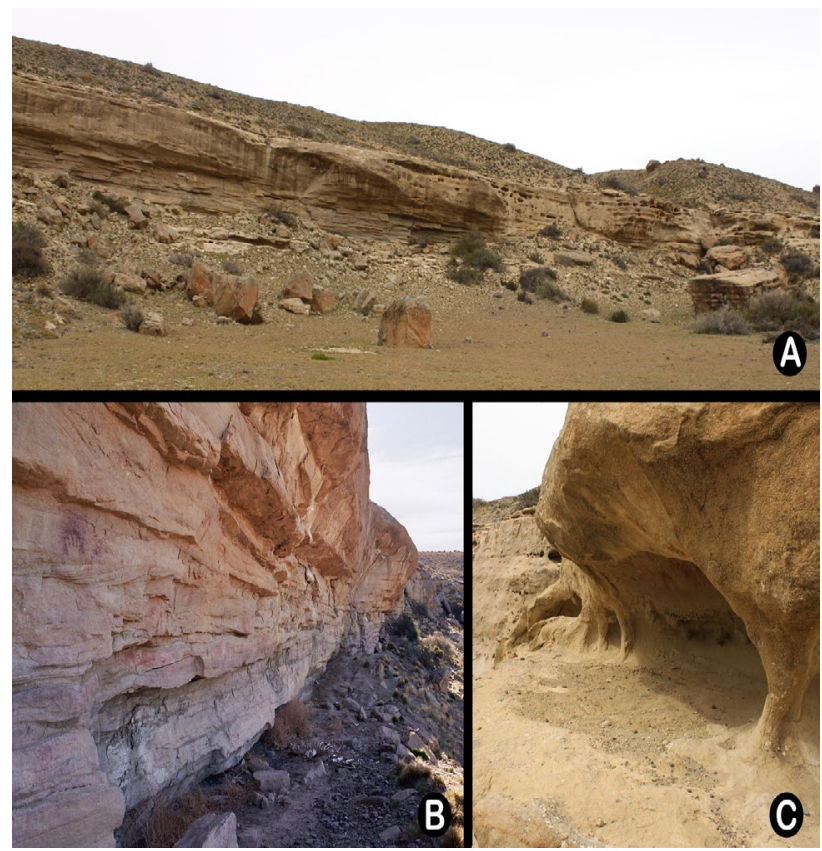

Figura 3. Alero con Manos del Río Chalía. A: Vista de todo el sitio, B: Sector 2, C: Nicho.

Figure 3. Alero con Manos del Río Chalía. A:View of all the site, B: Sector 2, C: Niche.

\section{Estado de conservación}

Se observaron diversos factores de deterioro que afectan a los motivos. Entre los naturales se cuenta en primer lugar el mismo soporte, una arenisca muy friable que se disgrega fácilmente, lo cual implica que muchas pinturas estén exfoliadas. Asimismo, se registraron otros daños naturales producto de la incidencia solar, caídas de agua, bloques desprendidos naturalmente y excremento de aves.

Las pinturas se encuentran en general desvaídas (52\%, $\mathrm{n}: 147)$ y muy desvaídas (30\%, n: 80), mientras que sólo un bajo porcentaje son claramente visibles (17\%, n: 57). Esto se relaciona con la exposición de los motivos a los elementos naturales, de tal manera que aquellas ubicadas en soportes más protegidos, como el caso del nicho, presentan un menor grado de desvaído. En función de ello, cabe mencionar que el tratamiento en laboratorio de las imágenes con el programa Images plugin DStretch prácticamente duplicó la cantidad motivos observada en el campo.

Por otra parte, se detectó la presencia de un total de 23

\begin{tabular}{ccccc}
\hline & & Largo $(\mathrm{m})$ & N Motivos & Densidad $(\mathrm{mot} / \mathrm{m})$ \\
\hline \multirow{4}{*}{ Alero } & Sector 1 & 11 & 49 & 4,45 \\
& Sector 2 & 18 & 113 & 6,28 \\
& Sector 3 & 9 & 48 & 5,33 \\
& Sector 4 & 8 & 54 & 6,75 \\
\cline { 2 - 5 } & Total & 50 & 264 & 5,28 \\
\hline Nicho & & 2,7 & 20 & 7,41 \\
\hline
\end{tabular}

Tabla 1. Distribución de motivos rupestres en los distintos sectores de AMRC. Referencias: mot.: motivo.

Table 1. Distribution of rock art motifs in the different sectors of AMRC. References: mot.: motif 


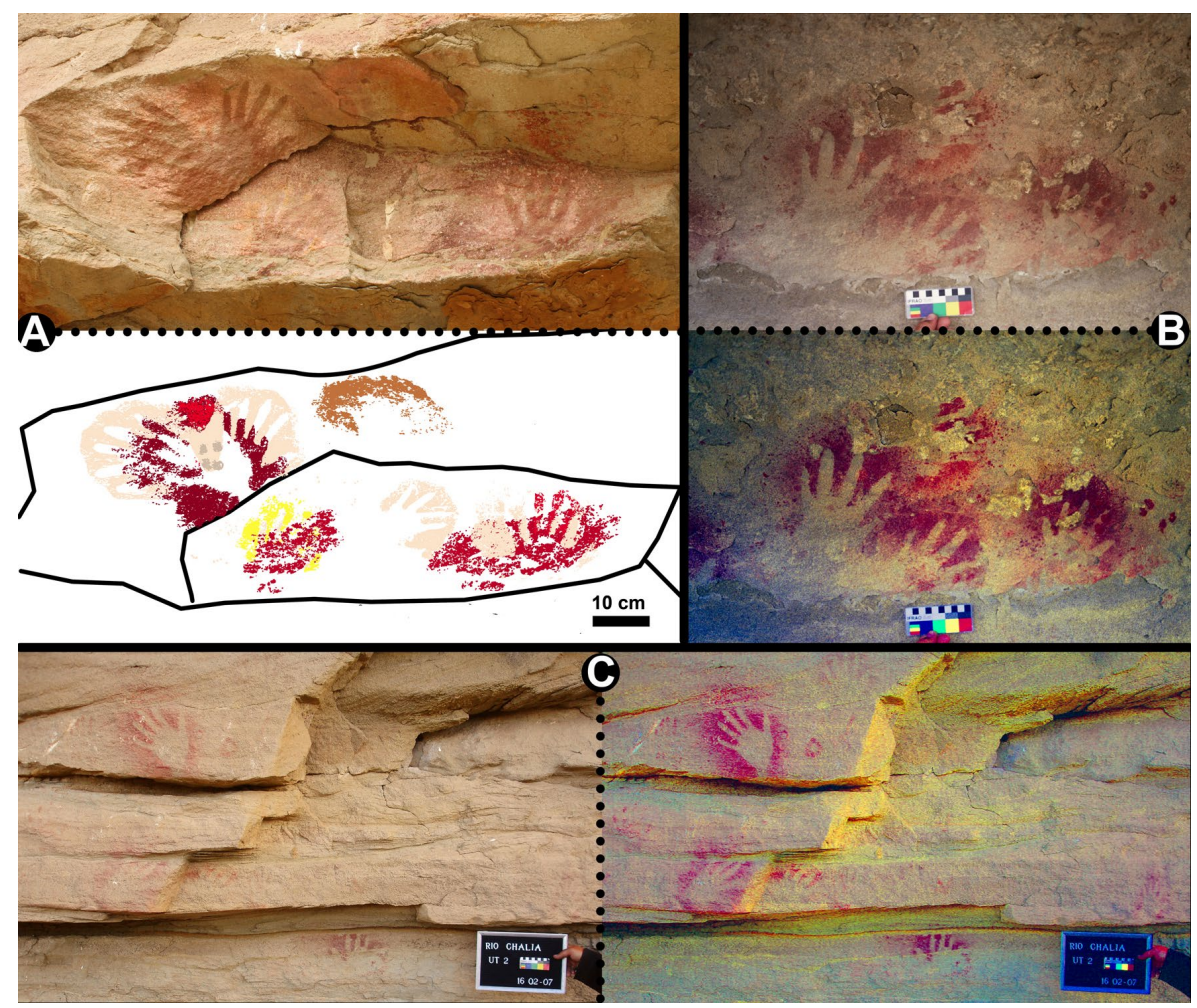

Figura 4. Pinturas de AMRC. A: Sector 4 (arriba: foto original, abajo: calco digital), B: Nicho (arriba: foto original, abajo: foto con filtro DStretch), C: Sector 2 (izquierda: foto original, derecha: foto con filtro DStretch).

Figure 4. Paintings in $A M R C$. A: Sector 4 (above: original photograph, down: digital tracing), B: Niche (above: original photograph, down: photograph with DStretch filter), C: Sector 2 (left: original photograph, right: photograph with DStretch filter).

daños antrópicos sobre las paredes del sitio. Nueve de ellos afectan directamente representaciones rupestres. La mayoría son graffitis, aunque también se observó un fake (una imitación) y un intento de sustracción de parte de la pared.

En función de los daños naturales y antrópicos que afectan a las representaciones, se estimó que el estado de conservación de las pinturas es principalmente regular $(41 \%, n: 115)$ y malo $(44 \%, n: 125)$.

Tipos de motivos

En el Alero con Manos del Río Chalía se identificaron 15 tipos de motivos (Tabla 2 y Figura 4). Dominan claramente los negativos de mano en un $65 \%$ (n: 184). Luego, los punteados (7\%, n: 20), los positivos de mano $(3,5 \%$, $\mathrm{n}: 10)$ y los trazos $(2,5 \%, \mathrm{n}: 7)$. Los restantes tipos de motivos son muy escasos, con menos de cinco $(<2 \%)$ cada uno. Entre ellos se cuentan abstractos como círculos, líneas curvas, líneas quebradas, líneas rectas, líneas sinuosas, subcirculares y triángulos. También se suman ejemplos únicos de tridígitos, huellas de felino y negativos de pata de guanaco, así como un negativo de objeto no identificado aproximadamente circular con dos protuberancias en la parte superior. Cabe mencionar que se registraron 44 motivos indeterminados por su

\begin{tabular}{lcccccccc}
\hline \multicolumn{1}{c}{ Tipo de motivo } & Sector 1 & Sector 2 & Alero & Sector 3 & Sector 4 & Total & Nicho & Total \\
\hline Negativo de mano & 38 & 64 & 33 & 32 & 167 & 17 & 184 \\
Positivo de mano & 2 & 5 & 2 & 1 & 10 & - & 10 \\
Huella de felino & - & - & - & 1 & 1 & - & 1 \\
Negativo de pata de guanaco & - & 1 & - & - & 1 & - & 1 \\
Tridígito & - & 1 & - & - & 1 & - & 1 \\
Círculo & 1 & 1 & - & - & 2 & - & 2 \\
Línea curva & - & 1 & - & - & 1 & - & 1 \\
Línea quebrada & 1 & 3 & - & 1 & 5 & - & 5 \\
Línea recta & - & - & 2 & - & 2 & - & 2 \\
Línea sinuosa & - & 2 & - & - & 2 & - & 2 \\
Punteados & 1 & 14 & 2 & 1 & 18 & 2 & 20 \\
Subcircular & 1 & 1 & - & - & 2 & - & 2 \\
Trazo & 1 & 5 & - & - & 6 & 1 & 7 \\
Triángulo & - & 1 & - & - & 1 & - & 1 \\
Negativo de objeto & - & - & - & 1 & 1 & - & 1 \\
Indeterminados & 4 & 14 & 9 & 17 & 44 & - & 44 \\
\hline Total & 49 & 113 & 48 & 54 & 264 & 20 & 284 \\
\hline
\end{tabular}

Tabla 2. Tipos de motivos presentes en AMRC.

Table 2. Motif types present in AMRC. 
Tabla 3. Tonalidades de los motivos en AMRC con detalle de su ubicación en los casos de superposiciones.

Table 3. Motifs colors in AMRC indicating its location in those cases that are superimposed.

\begin{tabular}{|c|c|c|c|c|c|c|}
\hline \multirow[b]{2}{*}{ Tonalidad } & \multirow[b]{2}{*}{$\begin{array}{l}\text { Posición } \\
\text { Inferior }\end{array}$} & \multicolumn{2}{|c|}{ Superpuestos } & \multirow[b]{2}{*}{$\begin{array}{c}\text { Orden no } \\
\text { determinado }\end{array}$} & \multirow{2}{*}{$\begin{array}{c}\text { Sin } \\
\text { superposición }\end{array}$} & \multirow[b]{2}{*}{ Total } \\
\hline & & $\begin{array}{l}\text { Posición } \\
\text { Superior }\end{array}$ & $\begin{array}{c}\text { Posición } \\
\text { Intermedia }\end{array}$ & & & \\
\hline Amarillo & 1 & 1 & & & 3 & 5 \\
\hline Blanco & & 9 & 3 & & 14 & 26 \\
\hline Celeste & 1 & & & & 2 & 3 \\
\hline Negro & 1 & & & & 1 & 2 \\
\hline Rojo & 9 & 10 & 3 & 10 & 112 & 144 \\
\hline Rojo anaranjado & & 3 & & & 11 & 14 \\
\hline Rojo violáceo & 11 & 3 & 2 & 2 & 55 & 73 \\
\hline Rosado & 3 & 1 & & & 1 & 5 \\
\hline Verde & & 1 & & & & 1 \\
\hline Violáceo & & & & 1 & 10 & 11 \\
\hline Total & 26 & 28 & 8 & 13 & 209 & 284 \\
\hline
\end{tabular}

estado de deterioro (15\%), con tres casos de manchas rectangulares que sugieren la figura de guanacos, aunque el estado de conservación no permite asegurarlo.

En relación a las manos (tanto negativas como positivas), en los casos donde se pudo determinar la lateralidad, más del $80 \%$ son izquierdas y de variados tamaños. Si bien a futuro hace falta realizar mediciones precisas que permitan considerar los grupos etarios (ver por ejemplo Carden y Blanco 2016, Guichon 2018), se identificaron al menos 18 manos pequeñas (10\%) que podrían asignarse a niños.

En la Tabla 2 puede asimismo observarse la distribución de los distintos tipos de motivos en los distintos sectores identificados en el sitio. En términos generales se observan tendencias similares en cuanto al predominio de los negativos de mano, seguidos por los positivos. Sin embargo, resalta la mayor variedad de motivos -particularmente abstractos- en el Sector 2, el cual es el de mayor extensión.

\section{Tonalidades y superposiciones}

Se reconocieron al menos diez tonalidades diferentes en las pinturas rupestres de AMRC (Tabla 3). La mayor parte de los motivos (50\%, n: 144) son rojos, siguen las representaciones rojo violáceas $(26 \%, \mathrm{n}: 73)$ y blancas (9\%, n: 26). En menores porcentajes, se detectaron otras tonalidades como rojo anaranjado (5\%, n: 14), violáceo $(4 \%, n: 11)$ y, en muy bajas frecuencias, amarillo, celeste, negro, rosado y verde'.

En función de los diversos factores de deterioro que afectan a las pinturas, se debe tener presente que los tonos que se observan actualmente en algunos motivos

\footnotetext{
${ }^{1}$ En la comparación con los colores documentados en otras áreas, llama la atención la presencia de escasos motivos en celeste y verde. A futuro se espera realizar análisis físico-químicos que permitan determinar si estos colores se deben al pigmento utilizado y/o a cuestiones de conservación.
}

no representan necesariamente los colores elegidos por quienes los elaboraron y probablemente se relacionen en parte con el tiempo transcurrido desde su ejecución. De esta manera, es posible comenzar a evaluar la presencia de distintos conjuntos tonales (sensu Aschero 1988), que permitan discriminar momentos de ejecución. Por supuesto, para discutir la secuencia relativa de ejecución, esta información debe ser complementada con aquella aportada por las superposiciones registradas.

En ese sentido, se identificaron 55 superposiciones, 44 de ellas en el alero propiamente dicho y 11 en el nicho. En la gran mayoría (n: 47) pudo determinarse el orden de ejecución de los motivos. Fueron registradas en todos los sectores del sitio aunque con mayor densidad en el Sector 4, donde se localizaron 20 casos.

Si se considera la ubicación de los motivos de las diversas tonalidades en las superposiciones (Tabla 3), se evidencian algunos patrones que cabe destacar. Por un lado, el rojo se halla en frecuencias similares tanto por encima como por debajo. Por otro lado, el rojo violáceo y el rosado se encuentran principalmente por debajo, mientras que el blanco se suele ubicar en posición superior.

\section{Secuencia de ejecución}

La presencia de superposiciones, distintas tonalidades y grados de desvaído sugieren varios momentos de ejecución en AMRC. Así, a partir de estos indicadores, se puede plantear una secuencia de producción de las pinturas rupestres. En primer lugar, se registrarían escasos motivos en rosado y celeste junto con representaciones rojo violáceo y posiblemente también violáceas. Posteriormente, se incorporarían las pinturas rojas y negras. Mientras que el uso de la tonalidad roja se prolongaría en el tiempo hasta el fin de la secuencia, la utilización del negro sería algo más acotado. Más adelante, se sumaría en dos momentos el empleo de la pintura blanca. Por último, se agregaría, aunque en bajas 
Tabla 4. Comparación con regiones cercanas a la cuenca de los lagos Tar y San Martín donde se registraron motivos rupestres.

Table 4. Comparison with nearby regions to the Tar and San Martin lakes basin where rock art was documented.

\begin{tabular}{|c|c|c|c|c|c|c|}
\hline & & $\begin{array}{c}\mathrm{N} \\
\text { sitios }\end{array}$ & $\begin{array}{c}\mathrm{N} \\
\text { motivos }\end{array}$ & Técnica & Soporte & Referencias \\
\hline \multirow{4}{*}{$\begin{array}{l}\text { Sectores } \\
\text { altos } \\
\text { (más de } 700 \\
\text { msnm) }\end{array}$} & $\begin{array}{l}\text { Mesetas de San } \\
\text { Adolfo y Cardiel Chico }\end{array}$ & 13 & $\begin{array}{c}798 \\
\text { (en } 8 \text { sitios) }\end{array}$ & Grabado & Basalto & $\begin{array}{l}\text { Belardi et al. 2009, 2013, } \\
\text { 2016b; Re et al. 2013-2014 }\end{array}$ \\
\hline & Meseta del Tobiano & 5 & $\begin{array}{c}\text { ND } \\
7429\end{array}$ & $\begin{array}{l}\text { Grabado } \\
\text { Grabado (+) }\end{array}$ & Basalto & Belardi et al. 2016b \\
\hline & Meseta del Strobel & 90 & (en 79 sitios) & y Pintura (-) & Basalto & Re 2010; Re et al. 2017 \\
\hline & La Criolla & 2 & ND & $\begin{array}{l}\text { Pintura y } \\
\text { Grabado }\end{array}$ & Basalto & Inédito \\
\hline \multirow{3}{*}{$\begin{array}{l}\text { Sectores } \\
\text { intermedios } \\
(400 \text { a } 700 \\
\text { msnm) }\end{array}$} & $\begin{array}{l}\text { Margen norte del lago } \\
\text { Viedma }\end{array}$ & 1 & ND & $\begin{array}{l}\text { Grabado (+) } \\
\text { y Pintura (-) }\end{array}$ & Arenisca & $\begin{array}{l}\text { Belardi et al. 2009, 2016b; } \\
\text { Menghin 1957; Schobinger y } \\
\text { Gradin } 1985\end{array}$ \\
\hline & $\begin{array}{l}\text { Nacientes del río } \\
\text { Chalía }\end{array}$ & 1 & ND & $\begin{array}{l}\text { Grabado (+) } \\
\text { y Pintura (-) }\end{array}$ & Arenisca & Belardi et al. 2016b \\
\hline & Laguna El Pajonal & 1 & ND & $\begin{array}{l}\text { Grabado (+) } \\
\text { y Pintura (-) }\end{array}$ & Arenisca & Belardi et al. 2016b \\
\hline \multirow{3}{*}{$\begin{array}{l}\text { Sectores } \\
\text { bajos } \\
(270 \text { a } 400 \\
\text { msnm) }\end{array}$} & $\begin{array}{l}\text { Cuenca de los lagos } \\
\text { Tar y San Martín }\end{array}$ & 2 & 285 & Pintura & Arenisca & Este trabajo \\
\hline & La Margarita & 2 & ND & $\begin{array}{l}\text { Grabado y } \\
\text { Pintura }\end{array}$ & Arenisca & Inédito \\
\hline & $\begin{array}{l}\text { Cuenca del lago } \\
\text { Cardiel }\end{array}$ & 22 & 1264 & $\begin{array}{l}\text { Pintura }(+) \text { y } \\
\text { Grabado }(-)\end{array}$ & $\begin{array}{l}\text { Arenisca y } \\
\text { Basalto }\end{array}$ & $\begin{array}{l}\text { Belardi y Goñi 2002; Ferraro y } \\
\text { Molinari 2010; Guichón 2016, } \\
\text { 2018; Re et al. } 2014\end{array}$ \\
\hline
\end{tabular}

frecuencias, una mayor variedad de tonalidades en las pinturas. Este sería el caso del amarillo, rojo anaranjado y verde.

Se propone que los negativos de manos se ejecutaron a lo largo de toda la secuencia. Presentan toda la variedad de tonalidades, gran cantidad de superposiciones y todos los grados de desvaído. Por otra parte, se sugiere que los motivos abstractos se realizaron tanto hacia el medio como al final de la secuencia. Estas representaciones se hicieron en rojo y rojo violáceo y en las superposiciones generalmente se encuentran en posición superior. Los positivos de mano se hallarían más hacia el final, ya que existen ejemplos en amarillo y verde y parecen estar menos desvaídos. El tridígito y la huella de felino también podrían haber sido elaborados hacia el fin de la secuencia, ya que fueron ejecutados en rojo y uno de ellos se encuentra arriba en una superposición.

Ahora bien, ¿qué cronología se le asigna a los distintos momentos de ejecución? En función de que la mayor cantidad de los fechados disponibles a nivel regional en la cuenca de los lagos Tar y San Martín y campos ubicados hacia el lago Viedma se ubican en los últimos 3000 años y en menor medida durante el Holoceno medio (Belardi et al. 2013a, 2016b), es probable que los motivos analizados fueran producidos a lo largo de varios momentos del Holoceno medio y tardío. Durante el Holoceno temprano se ha propuesto una baja densidad de ocupaciones restringida al sector del lago San Martín y por el momento no se postula la ejecución de pinturas rupestres durante este período. Se evidencia la necesidad de obtener en futuros trabajos fechados en el Alero con Manos del Río Chalía y contextualizar las representaciones con otras líneas de evidencia a fin de corroborar la cronología sugerida.

\section{Las pinturas de la cuenca en contexto macrorregional}

En este punto resulta de utilidad considerar la información sobre otros sitios con representaciones rupestres que se localizan en espacios cercanos (Figura 1). De esta manera, se busca evaluar el papel que cumplió la cuenca bajo estudio en la circulación de información a través de medios materiales. Para facilitar la comparación los datos disponibles son organizados de acuerdo a los sectores altitudinales definidos previamente para los lagos Tar y San Martín (Tabla 4).

En los sectores por encima de los 700 msnm linderos con la cuenca, como las mesetas de San Adolfo y Cardiel Chico al norte (Belardi et al. 2009, 2013b, 2016b; Re et al. 2013-2014) y la meseta del Tobiano al sur (Belardi et al. 2016b), se registraron exclusivamente motivos grabados en paredones de basalto. Se documentaron una mayor cantidad de sitios con representaciones de diversas morfologías y tamaños. Se propuso una cronología tardía para la mayoría de ellos a partir de las evidencias asociadas y las características de los grabados, con un menor porcentaje correspondiente al Holoceno medio. Por su parte y, como fuera mencionado, hacia el noreste destaca el sitio La Criolla 1. Si bien presenta una baja frecuencia total de motivos ejecutados sobre paredones basálticos, registra tanto pinturas de diversas tonalidades como grabados.

Hacia el sur de la cuenca, en los sectores intermedios (400 a $700 \mathrm{msnm}$ ), se documentaron tres sitios con motivos rupestres ubicados en la margen norte del lago Viedma, en las nacientes del Chalía y en las cercanías de la Laguna El Pajonal (Belardi et al. 2009, 2016b; Menghin 1957; Schobinger y Gradin 1985). Se caracterizan por presentar una mayor cantidad de grabados y menor frecuencia de 
pinturas y localizarse sobre farallones de arenisca. En particular, se ha destacado Punta del Lago Viedma 1, por la cantidad y diversidad de motivos que presenta (Belardi et al 2009; Menghin 1957; Schobinger y Gradin 1985). Los restantes sitios que se han sumado recientemente son La Zulema en las nacientes del río Chalía y Laguna El Pajonal 1 (Belardi et al. 2016b). Los fechados disponibles y los diseños de las representaciones sugieren ocupaciones concentradas en los últimos 3000 años y algunos eventos datados durante el Holoceno medio.

En sectores bajos (270 a $400 \mathrm{msnm}$ ) se han localizado recientemente dos sitios con representaciones rupestres en la Ea. La Margarita, hacia el este de la región de estudio, sobre un tributario del arroyo Los Paisanos. El más cercano es Cueva La Margarita, que se encuentra $17 \mathrm{~km}$ al sureste del sitio AMRC y, si bien es pequeña $(2,7 \mathrm{~m}$ de profundidad y 4,5 $\mathrm{m}$ de ancho), presenta muchas similitudes con dicho alero en términos del uso exclusivo de la pintura, el predomino de negativos de manos y presencia de diversas tonalidades con una similar secuencia de ejecución. El otro sitio se denomina Farallón La Margarita, se encuentra $3 \mathrm{~km}$ al suroeste de la cueva y tiene una extensión aproximada de $500 \mathrm{~m}$. Exhibe solamente grabados, aunque con una diversidad de tipos de motivos mucho mayor que la cueva registrada en la misma estancia. Cabe destacar que su análisis preliminar indica que los tipos de motivos representados mediante grabado en el farallón se distinguen de aquellos ejecutados con la misma técnica en las mesetas altas, lo cual marca una diferenciación que a futuro será interesante explorar.

La información de los diversos espacios evidencia una mayor importancia de las mesetas altas y luego de los sectores intermedios para la comunicación a través de las representaciones rupestres. En estos se observa una mayor cantidad de sitios, con diversidad de tipos de motivos y con un uso casi exclusivo del grabado. Dejando de lado los sectores bajos analizados en este trabajo, las pinturas sólo se encuentran en menores porcentajes en los espacios intermedios en sitios como La Zulema y Laguna El Pajonal 1 y, en los espacios altos, en La Criolla. De esta manera, la comparación realizada permite mostrar que el uso de la pintura y la presencia de negativos de mano destacan en los sectores bajos y particularmente en AMRC.

Al ampliar la escala espacial, es importante considerar la información proveniente de la cuenca bajo estudio en comparación con los patrones identificados en la cuenca del lago Cardiel y la colindante meseta del lago Strobel, ubicadas a una distancia lineal de $60 \mathrm{~km}$ al norte de AMRC. En primer lugar, en la cuenca del lago Cardiel se identificaron 22 sitios de distintos tamaños con más de 1200 motivos (Guichon 2018). La mayor cantidad de representaciones se realizaron mediante pintura con un muy bajo porcentaje de grabado. Los soportes utilizados fueron tanto areniscas como basaltos. Se evidencian varias similitudes con la cuenca de los lagos Tar y San Martín, principalmente con el sitio AMRC en el curso superior del río Chalía: presencia de los mismos tipos de motivos, el uso casi exclusivo de la pintura y diversidad de tonalidades. No obstante, también existen diferencias, ya que en el lago Cardiel se documentó una distribución más amplia de sitios con pinturas, coincidente con una mayor disponibilidad de soportes potenciales, y una mayor diversidad de tipos de motivos en algunos sitios. Es interesante destacar que la secuencia de ejecución propuesta para la cuenca del lago Cardiel (Guichon 2018; Re et al. 2014) es coherente con la sugerida para el sitio AMRC. En ese sentido, se planteó que, si bien habría un menor porcentaje de pinturas que podría asignarse al Holoceno medio, la mayor parte de las representaciones del lago Cardiel datarían de momentos tardíos. Por otra parte, en la meseta del lago Strobel (700 a 1200 msnm) se documentaron 90 sitios con más de 7000 motivos (Re et al. 2017). La gran mayoría son grabados y se registró una enorme variedad de diseños. La ejecución de las representaciones fue asignada principalmente a los últimos 2500 años con una menor frecuencia realizada entre 5000 y 2500 años atrás. De esta manera, los sitios de la meseta del Strobel presentan similitudes con aquellos registrados en las de San Adolfo, Cardiel Chico y del Tobiano.

\section{Discusión y conclusiones}

Al evaluar la distribución de las representaciones rupestres hacia el interior de los sectores bajos asociados a la cuenca de los lagos Tar y San Martín, se hace evidente una baja densidad de sitios con motivos rupestres, ya que sólo se detectaron dos de ellos. Esto podría responder a diversas razones, más allá de las conductas de los grupos humanos en el pasado, tales como problemas de preservación, baja visibilidad de los motivos y escasa oferta de soportes potenciales. No obstante, si bien éstas podrían haber contribuido, existen soportes potenciales cercanos al lago San Martín, algunos con concentraciones de materiales líticos asociados, que no presentan motivos rupestres. En ese sentido, el hallazgo de un único motivo en el Bloque 1 del Campo de Bloques 1 en la margen sureste del lago es coherente con el uso menos intenso propuesto para este sector de la cuenca (Belardi et al. 2010, 2013a).

Por otra parte, en la margen este del lago Tar y en los alrededores del Cerro Cach Aike, donde se sugirió un uso residencial y más redundante (Belardi et al. 2010, 2013a), el único soporte potencial se registró sobre el lado este del Cerro Cach Aike, donde se documentó un bloque con un alero con materiales líticos pero sin presencia de motivos rupestres. Cabe mencionar el hallazgo de una placa grabada y cerámica decorada (Belcaguy 1983; Molinari 1983) en sitios a cielo abierto sobre el oeste del Cerro, los cuales podrían ser indicadores de otros medios materiales de comunicación. 
Otro aspecto destacado de los espacios bajos estudiados refiere a una importante diferenciación interna, ya que los dos sitios con motivos rupestres son claramente contrastantes. El sitio Alero con Manos del Río Chalía posee la mayor cantidad y diversidad de pinturas rupestres del sector bajo. Esto apunta a una mayor jerarquía de la cuenca superior del río Chalía desde el punto de vista de las representaciones y por tanto de la comunicación mediante este medio. Como se mencionó, el alero se encuentra en directa relación con una vía de circulación natural que conecta estas cuencas con espacios hacia el este (Belardi et al. 2009).

En trabajos anteriores ya se había planteado que la presencia de obsidiana, limolita y de moluscos marinos inscribe a la cuenca bajo estudio en una dinámica de interacción y circulación de bienes en una escala macrorregional (Belardi et al. 2009; Espinosa et al. 2007). Asimismo, las similitudes de los grabados rupestres en espacios altos colindantes como las mesetas de San Adolfo y Cardiel Chico y la del Tobiano con las representaciones de la meseta del Strobel, ubicada más al norte, apuntan también a la circulación de información por este medio (Belardi et al. 2009, 2013b; Re et al. 2013-2014).

A partir de estas evidencias, se propuso la articulación de la cuenca de los lagos Tar y San Martín con las de los lagos Cardiel y Viedma, ubicadas hacia el norte y sur respectivamente (Belardi et al. 2009). Así, se postularon tres rutas naturales de uso complementario. Dos de ellas serían de uso estacional dado que se transita por espacios altos. En particular, se planteó que la evidencia arqueológica sostenía la ruta que discurre por las meseta del Cardiel Chico, el valle del río Tar, la margen este del lago Tar, el río Meseta y luego el río Cangrejo hasta alcanzar la margen norte del lago Viedma². La tercera ruta, que implica la circulación a través del valle del río Chalía estaría disponible la mayor parte del año. Cuando se realizó la propuesta, se indicaba la falta de información arqueológica para poder evaluarla (Belardi et al. 2009). Así, el análisis realizado sobre las pinturas rupestres de los sectores bajos empiezan a llenar el vacío y apunta a la relevancia de este acceso a los lagos Tar y San Martín a la vez que se complementa la discusión sobre la interacción y circulación de información es una escala espacial amplia. De forma preliminar, los motivos grabados registrados hacia el sureste de la región en el sitio Farallón La Margarita, también en sectores bajos, apunta a una mayor complejidad en la comunicación a través de este medio que deberá continuar siendo evaluada.

Por último, la comparación de los dos sitios considerados con la información disponible sobre representaciones rupestres en regiones cercanas permite discutir el papel de los espacios bajos asociados a la cuenca de los lagos

\footnotetext{
${ }^{2}$ Trabajos recientes también mostraron la posibilidad de la utilización de la laguna El Pajonal durante el tramo medio del recorrido de esta ruta (Belardi et al. 2016b).
}

Tar y San Martín en un contexto macro-regional. En primer lugar, apunta a un uso complementario de los sectores bajos, intermedios y altos e indica la circulación de información de distinto tipo y con diferente intensidad en cada uno de ellos. En las mesetas se presenta una mayor frecuencia, densidad y diversidad de motivos que en los espacios bajos. En estos términos, destaca una mayor jerarquía de los sectores altos e intermedios, al menos durante el Holoceno tardío, lo que es concordante con el modelo de convergencia propuesto para la meseta del Strobel (Belardi y Goñi 2006; Re 2010) y que parece ser relevante para discutir las mesetas en general.

A la vez, en dicha meseta y en la cuenca del lago Cardiel también se verifica un uso complementario de espacios altos y bajos (Belardi y Goñi 2002; Guichon 2018). Esto sugiere una estrategia de circulación de información muy semejante al menos durante el Holoceno tardío. Por otra parte, si se contrasta la información proveniente solamente de las dos cuencas bajas, se evidencia una mayor comunicación a través de las representaciones rupestres en la cuenca del lago Cardiel que en la de los lagos Tar y San Martín. Así, la información presentada apoya la propuesta de que el patrón de ocupación de esta última estaría desacoplado del de la cuenca del lago Cardiel y que, particularmente durante momentos tardíos, habría sido utilizada de manera menos intensa (Belardi et al. 2013a).

La discusión de los sitios con pinturas rupestres en sectores bajos asociados a la cuenca de los lagos Tar y San Martín permite completar así un panorama que se presenta cada vez más complejo en relación a la comunicación vía medios materiales. En ese sentido, destacan por un lado las diferencias entre espacios altos y bajos y por otro la jerarquización de sitios al interior de cada uno de ellos. De esta manera, se profundiza la comprensión de los mecanismos de circulación de información empleados en el centro-oeste del extremo sur de Patagonia, principalmente durante el Holoceno tardío.

Buenos Aires, 27 de diciembre de 2017

\section{Agradecimientos}

Agradecemos especialmente a quienes colaboraron en los relevamientos de los sitios analizados: Augusto Tessone, Francisco Guichon, Lumila Menéndez, Flavia Carballo Marina, Silvana Espinosa, Amalia Nuevo Delaunay y Sergio Raggi. A los dueños y personal de las Eas. Río Meseta, Lago Tar, Santa Margarita, Punta del Lago y La Margarita por su gentileza y ayuda durante los trabajos de campo. A Daniel Grima por la confección del mapa. También agradecemos los oportunos comentarios de los evaluadores, de Flavia Carballo Marina y de Alejandro Súnico. Las investigaciones están siendo subsidiadas por los proyectos UNPA 29/A304-1 y 29/A360-1 y PICT 20160373. 


\section{Bibliografía}

Aschero, C. A. (1988). Pinturas rupestres, actividades y recursos naturales: un encuadre arqueológico. En H. Yacobaccio (Ed.), Arqueología Contemporánea Argentina. Actualidad y Perspectivas (pp. 109-145). Buenos Aires: Ediciones Búsqueda.

Aunger, R. (2009). Human Communication as Niche Construction. En S. Shennan (Ed.), Pattern and Process in Cultural Evolution (pp. 33-43). Berkeley: University of California Press.

Bamonte, F. P, M. V. Mancini, J. B. Belardi y S. Espinosa. (2013). Inferencias paleoambientales a partir del análisis polínico de sitios arqueológicos del área del lago San Martín (Santa Cruz, Argentina). Magallania, 41 (1), 155-169.

Barton, C. M., G. A. Clark y A. E. Cohen. (1994). Art as information: explaining Upper Paleolithic art in Western Europe. World Archaeology, 26 (2), 185-207.

Belardi, J. B. y P. Campan. (2009). Resultados arqueológicos en el Campo de Bloques 2, margen sur del lago San Martín (Provincia de Santa Cruz). En M. Salemme, F. Santiago, M. Alvarez, E. Piana, M. Vázquez y E. Mansur (Eds.), Arqueología de la Patagonia: una mirada desde el último confín, Tomo 2 (pp. 927-939). Ushuaia: Editorial Utopías.

Belardi, J. B. y F. Carballo Marina. (2014). La señal arqueológica del interior del bosque en la margen sur del lago San Martín (Provincia de Santa Cruz). Comechingonia, 18 (2), 181-202.

Belardi, J. B. y R. A. Goñi. (2002). Distribución espacial de motivos rupestres en la cuenca del lago Cardiel (Patagonia Argentina). Boletín SIARB, 16, 29-38.

Belardi, J. B. y R. A. Goñi. (2006). Representaciones rupestres y convergencia poblacional durante momentos tardíos en Santa Cruz (Patagonia argentina). El caso de la meseta del Strobel. En D. Fiore y M. M. Podestá (Eds.), Tramas en la Piedra. Producción y usos del arte rupestre (pp. 85-94). Buenos Aires: World Archaeological Congress, Sociedad Argentina de Antropología y Asociación Amigos del Instituto Nacional de Antropología.

Belardi, J. B., F. Carballo Marina, T. J. Bourlot y A. Re. (2009). Paisajes arqueológicos, circulación e interacción en diferentes escalas: una perspectiva desde el lago Tar (Provincia de Santa Cruz). En M. Salemme, F. Santiago, M. Álvarez, E. Piana, M. Vázquez y E. Mansur (Eds.), Arqueología de la Patagonia: una mirada desde el último confín (pp. 219-231). Ushuaia: Editorial Utopías.

Belardi, J. B., S. Espinosa, F. Carballo Marina, G. Barrientos, R. A. Goñi, A. Súnico, T. Bourlot, C. Pallo, A. Tessone, S.
García Guraieb, A. Re y P. Campan. (2010). Las cuencas de los lagos Tar y San Martín (Santa Cruz, Argentina) y la dinámica del poblamiento humano del sur de Patagonia: integración de los primeros resultados. Magallania, 38 (2), 137-159.

Belardi, J. B., G. Barrientos, F. Bamonte, S. Espinosa y R. Goñi. (2013a). Paleoambientes y cronología de las ocupaciones cazadoras recolectoras de las cuencas de los lagos Tar y San Martín (provincia de Santa Cruz). Intersecciones en Antropología, 14, 459-475.

Belardi, J. B., S. Espinosa, G. Barrientos, F. Carballo Marina, A. Re, P. Campan, A. Súnico y F. Guichón. (2013b). Las mesetas de San Adolfo y Cardiel Chico: estrategias de movilidad y tácticas de caza de guanacos en el SO de Santa Cruz. En F. Zangrando, R. Barberena, A. Gil. G. Neme, M. Giardina, L. Luna, C. Otaola, L. Paulides, L. Salgan y A. Tívoli (Eds), Tendencias teórico-metodológicas y casos de estudio en la arqueología de Patagonia (pp. 261-270). Buenos Aires: Museo de Historia Natural de San Rafael - SAA - INAPL.

Belardi, J. B., G. Cassiodoro, R. A. Goñi, M. Glascock y A. Súnico. (2015). Siltstone from Southern Patagonia: Its source and Archaeological Artifact Distribution in Santa Cruz Province, Argentina. Geoarchaeology: An International Journal, 30, 223-237.

Belardi, J. B., R. Barberena, R. Goñi y A. Re. (2016a). The Development of a Legacy: Evolution, Biogeography and Archaeological Landscapes. En M. Cardillo y H. Muscio (Eds.), Darwin's Legacy: The Status of Evolutionary Archaeology in Argentina, (pp. 83-94). Oxford: Archaeopress.

Belardi, J. B., S. Espinosa, F. Carballo Marina, G. Barrientos, P. Campan y A. Súnico. (2016b). Desde la meseta del Cardiel Chico a la margen norte del lago Viedma (provincia de Santa Cruz, Argentina): nuevos datos sobre el paisaje arqueológico. En F. Mena (Ed), Arqueología de Patagonia: de Mar a Mar (pp. 411-420). Santiago de Chile: Ediciones CIEP-Ñire Negro.

Belçaguy, H. (1983). Tipología de los materiales líticos del médano Estancia Cach-Aike - colección Thierauf sitios 31, 48 y 49 - lago San Martín - provincia de Santa Cruz (Tesis de Licenciatura inédita). Facultad de Filosofía y Letras, Universidad de Buenos Aires, Buenos Aires.

Borrero, L. A. (2004). The Archaeozoology of Andean 'Dead Ends' in Patagonia: Living near the Continental Ice Cap. En M. Mondini, A. S. Nuñoz y S. Wickler (Eds.), Colonisation, Migration and Marginal Areas. A Zooarchaeological approach, (55-61). Oakville: Oxbow Books.

Carden, N y R. Blanco. 2016. Measurements and 
Replications of Hand Stencils: a Methodological Approach for the Estimation of the Individuals' Age and Sex. En R. Bednarik, D. Fiore, M. Basile, T. Huisheng y G. Kumar (Eds), Palaeoart and Materiality: The Scientific Study of Rock Art (pp. 129-146). Oxford: Archaeopress Publishing Ltd.

Espinosa, S. L., J. B. Belardi y R. Molinari. (2007). Análisis tecnológico de los artefactos líticos de la colección Horst Thierauf provenientes de las cuencas de los lagos Tar y San Martín (Provincia de Santa Cruz, Argentina). En F. Morello, M. Martinic, A. Prieto y G. Bahamonde (Eds), Arqueología de Fuego-Patagonia. Levantando piedras, desenterrando huesos... y develando arcanos (pp. 675685). Punta Arenas: Ediciones CEQUA.

Espinosa, S. L., J. B. Belardi, G. Barrientos y F. Carballo Marina. (2013). Poblamiento e intensidad de uso del espacio en la cuenca del lago San Martin (Patagonia argentina): nuevos datos desde la margen norte. Comechingonia, 17 (1), 105-121.

Ferraro, L. y R. Molinari. (2010). ¡Último momento! El arte de los cazadores-recolectores recorre el lago Cardiel y se dirige al Strobel! En F. Oliva, N. de Grandis y J. Rodriguez (Comp) Arqueología Argentina en los Inicios de un Nuevo Siglo. Publicación del XIV Congreso Nacional de Arqueología Argentina, Tomo III (pp. 523-534). Rosario: Centro Estudios Arqueológicos-Facultad de Humanidades y Artes-Universidad Nacional de Rosario.

Guichon, F. (2016). Representaciones rupestres en los Cañadones de la cuenca del Lago Cardiel: Alero del León y sitios aledaños (Santa Cruz). En F. Mena (Ed), Arqueología de Patagonia: de Mar a Mar (pp. 450-459). Santiago de Chile: Ediciones CIEP-Ñire Negro.

Guichon, F. (2018). Comunicación y circulación de información entre espacios altos y bajos en Patagonia Meridional: Representaciones rupestres del lago Cardiel y la meseta del Strobel (Tesis de doctorado). Facultad de Filosofía y Letras, Universidad de Buenos Aires, Buenos Aires.

McDonald, J. y P. Veth. (2011). Information exchange among Hunter-Gatherers of the western desert of Australia. En R Whallon, W. A. Lovis y R. Hitchcock (Eds.), Information and its Role in Hunter-Gatherer Bands. (pp. 221-233). Los Angeles: UCLA Cotsen Institute of Archaeology Press.

Menghin, O. (1957). Estilos del arte rupestre de Patagonia. Acta Praehistorica, 1, 57-87.
Molinari, R. (1983). Tipología de los materiales líticos de Ea. Cach-Aike, Colección Thierauf, Lago San Martín, Provincia de Santa Cruz (Tesis de Licenciatura inédita). Facultad de Filosofía y Letras, Universidad de Buenos Aires, Buenos Aires.

Odess, D. (1998). The Archaeology of Interaction: Views from Artifact Style and Material Exchange in Dorset Society. American Antiquity 63 (3), 417-435.

Pallo, C. (2009). La tecnología lítica del sitio Bloque 1-Oquedad (lago San Martín, Provincia de Santa Cruz). Resultados de la primera etapa de análisis. En M. Salemme, F. Santiago, M. Alvarez, E. Piana, M. Vázquez y E. Mansur (Eds), Arqueología de la Patagonia: una mirada desde el último confín, Tomo 1 (pp. 369-381). Ushuaia: Editorial Utopías.

Rabassa, J. y A. Coronato. (2002). Glaciaciones del Cenozoico tardío. En M. Haller (Ed), Geología y recursos naturales de Santa Cruz. (pp. 303-315). Relatorio del CXV Congreso Geológico Argentino, Buenos Aires.

Re, A. (2010). Representaciones Rupestres en Mesetas Altas de la Provincia de Santa Cruz. Circulación de Información en Espacios de Uso Estacional (Tesis de doctorado inédita). Facultad de Filosofía y Letras, Universidad de Buenos Aires, Buenos Aires.

Re, A., F. Guichón y J. B. Belardi. (2013-2014). Las mesetas de San Adolfo y del Cardiel Chico (provincia de Santa Cruz): su uso y jerarquización regional a partir de los motivos rupestres. Cuadernos del Instituto Nacional de Antropología y Pensamiento Latinoamericano, 23 (2), 91-106.

Re, A., L. Ferraro, F. Guichón y R. Molinari. (2014). Las representaciones rupestres de la cuenca del lago Cardiel. En R. Goñi, J. B. Belardi, G. Cassiodoro y A. Re (Eds), Arqueología de las cuencas de los lagos Cardiel y Strobel. Poblamiento humano y paleoambientes en Patagonia (pp. 135-154). Buenos Aires: Aspha Ediciones.

Re, A., R. Goñi, J. Flores Coni, F. Guichón, J. Dellepiane y M. Umaño. (2017). Arqueología de la meseta del Strobel (Patagonia meridional): 15 años después. Relaciones de la Sociedad Argentina de Antropología, XLII (1), 133-158.

Schobinger, J. y C. Gradin. (1985). Arte rupestre de la Argentina. Cazadores de la Patagonia y agricultores andinos. Madrid: Encuentro Ediciones. 